\title{
Gettering Efficacy of an APCVD Glasses Based Stacked Co-Diffusion for Bifacial mc-Si PERT Solar Cells
}

\author{
Johannes Fichtner ${ }^{\mathrm{a})}$, Annika Zuschlag ${ }^{\mathrm{b})}$, and Giso Hahn ${ }^{\mathrm{c})}$ \\ Department of Physics, University of Konstanz, Universitaetsstrasse 10, 78457 Konstanz, Germany \\ a)Corresponding author: johannes.fichtner@uni-konstanz.de \\ b)annika.zuschlag@uni-konstanz.de \\ c)giso.hahn@uni-konstanz.de
}

\begin{abstract}
Gettering of impurities is an important task in p-type mc-Si solar cell production. Phosphorus diffusion via $\mathrm{POCl}_{3}$ is currently the most common way to achieve this. We report about the gettering efficacy of a co-diffusion in which $\mathrm{POCl}_{3}$ is not used. Phosphorus and boron containing glasses are both deposited prior to the diffusion via atmospheric pressure chemical vapor deposition (APCVD). The presented APCVD glasses based co-diffusion allows a loading without spacing and therefore has the potential for a very high throughput. As we demonstrate in this work, the overall gettering efficacy in different kinds of stacks is similar to the gettering efficacy in non-stacked APCVD glasses based diffusions. The reduction of interstitial iron in the analyzed lifetime samples is ascribed to phosphorus diffusion gettering and works in stacks just as well.
\end{abstract}

\section{INTRODUCTION}

The ambitious effort for ever increasing efficiencies and decreasing costs demands sophisticated approaches for future solar cell production concepts. Solar cell designs which allow for higher efficiencies than standard Aluminum back-surface-field (Al-BSF) cells usually require more process steps which in turn cause higher production costs. Key element in the technological evolution of Al-BSF silicon solar cells is the reduction of recombination losses, in particular at the rear side. PERC (passivated emitter and rear cell) solar cells for example benefit from passivated rear sides [1]. Reduction of recombination losses at the back is also possible with a fully diffused BSF, which is a feature of PERT (passivated emitter, rear totally diffused) solar cells. In addition, full metallization of the rear may be dispensed and a bifacial use of the solar cells is possible. Though, the production of this kind of solar cells normally requires several additional steps compared to Al-BSF or PERC solar cells.

The PERT approach we present aims for a simple and efficient production process which is meant to be used with cost-efficient p-type mc-Si material. One of the key features of the process is the use of atmospheric pressure chemical vapor deposition (APCVD) technique for fast and unilateral deposition of boron / phosphorus containing silicate glasses (BSG/PSG) prior to diffusion. As BSG and PSG are deposited via APCVD, neither $\mathrm{POCl}_{3}$ nor $\mathrm{BBr}_{3} / \mathrm{BCl}_{3}$ gas flows are necessary during the (co-)diffusion in which emitter and BSF are formed simultaneously. Since the dopant containing glasses are already deposited before diffusion, wafer spacing is not compulsory required which means wafers can be loaded by stacking. The described stacked co-diffusion has the potential to significantly decrease time and energy consumption for emitter and BSF diffusion in PERT solar cell production.

However, p-type mc-Si features a wide variety of (metal) impurities which makes efficient gettering inevitable. Efficient gettering in APCVD glasses based co-diffusion works in principle and the process is suitable for PERT solar cell production [2]. Though, the thermal load of wafers at different positions in a stacked co-diffusion may differ among each other and from standard co-diffusions. Therefore, the first point to be clarified is the gettering efficacy which is the focus of this work. Solar cell precursors, diffused in different kinds of stacks are compared to reference samples co-diffused in single slots in terms of effective minority charge carrier lifetime $\tau_{\text {eff }}$ and interstitial iron concentration $\left[\mathrm{Fe}_{\mathrm{i}}\right]$. 


\section{EXPERIMENTAL APPROACH}

In order to verify the feasibility of the stacked co-diffusion process with regard to gettering, lifetime samples were fabricated. The samples presented were chosen from an industrial boron-doped mc-Si G5 ingot $(1 \Omega \mathrm{cm})$ and have an initial thickness of $180 \mu \mathrm{m}$. All wafers were taken from vertically adjacent positions (sister wafer) to provide similar grain and defect structures. The process sequence for the lifetime samples (Fig. 1) is similar to the targeted PERT solar cell process and includes all steps which could influence the impurity concentrations and distributions.

After laser cutting into $50 \times 50 \mathrm{~mm}^{2}$ pieces the saw damage was etched off. The subsequent BSG / PSG deposition steps were made with an APCVD system from SierraTherm. The wafers are transported on a belt in horizontal position which allows single sided depositions. The BSG was deposited on the back side and capped with $\mathrm{SiO}_{\mathrm{y}}: \mathrm{H}$ in one pass. In a second APCVD pass $\mathrm{SiO}_{\mathrm{y}}: \mathrm{H}$ capped PSG was deposited on the front side. The subsequent co-diffusions were carried out in a tube furnace with standard spacing, in vertical stacks and in horizontal stacks. For the vertically stacked co-diffusion, four lifetime samples were placed in one slot of a usual quartz glass boat which allows an up to fourfold higher throughput without any modifications of the furnace. To perform a horizontally stacked co-diffusion, dummy wafers were used to keep a stack of 11 lifetime samples in place. The sheet resistance resulting from the applied diffusion was measured on equally produced p-type Czochralski grown silicon via fourpoint probing. The boron BSF has $\mathrm{R}_{\text {sheet }}=234 \Omega / \square$ and the phosphorus emitter $\mathrm{R}_{\text {sheet }} 64 \Omega / \square$.

After the diffusions, $\mathrm{SiO}_{\mathrm{y}}: \mathrm{H}, \mathrm{BSG}$ and $\mathrm{PSG}$ were removed in hydrofluoric acid. In order to remove emitter and BSF, $10 \mu \mathrm{m}$ of each side were etched off afterwards. $\mathrm{SiN}_{\mathrm{x}}: \mathrm{H}$ surface passivation was done with plasma-enhanced chemical vapour deposition (PECVD) on both sides. Finally, all lifetime samples were fired at $850^{\circ} \mathrm{C}$ peak set temperature. The gettering efficacy was evaluated via time resolved photoluminescence imaging (TR-PLI) [3] for spatially resolved $\tau_{\text {eff }}$ information and via quasi-steady-state photoconductance decay (QSSPC) measurements in combination with normal photoluminescence imaging (PLI) for spatially resolved [ $\mathrm{Fe}_{\mathrm{i}}$ ] information [4-6]. The QSSPC measurements were performed with a Sinton WCT-120 lifetime tester.

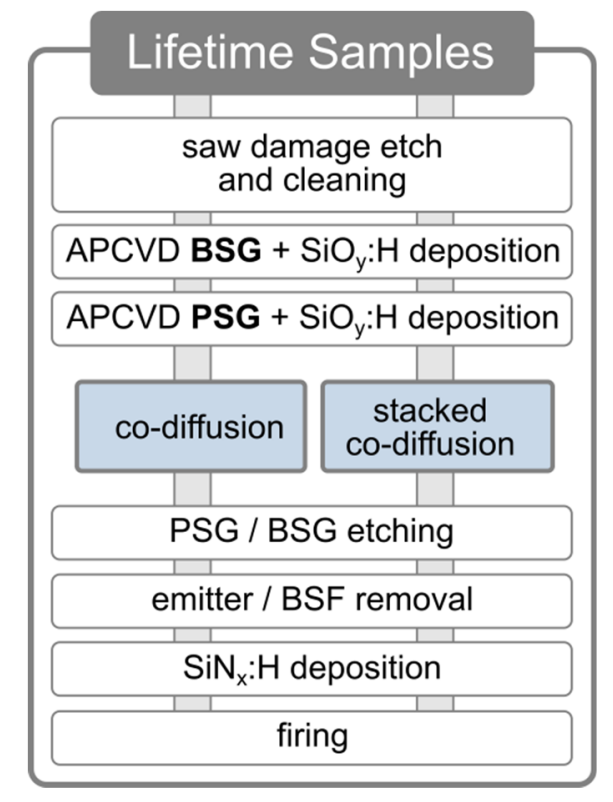

FIGURE. 1. Process flow chart for APCVD glasses based co-diffused lifetime samples. The co-diffusion for part of the samples was performed in different kind of stacks.

\section{RESULTS AND DISCUSSION}

One of the lifetime samples was only saw damage etched, cleaned and passivated. This sample serves as reference for the gettering efficacy of the co-diffusions. Another (sister) lifetime sample was co-diffused in vertical 
position without stacking (as the standard co-diffusion is usually performed). $\tau_{\text {eff }}$ increased by more than $90 \mu$ s compared to the reference sample (Fig. 1). This gives an indication for the gettering efficacy of the standard APCVD glasses based co-diffusion. The diffusion without stacking in horizontal position was similarly effective. The reason for the marginally lower improvement could be a slightly different thermal history because of the horizontal position, yet could also be owed by small variations in sample processing or the mc-Si material. However, the horizontal co-diffusion shows a significant gettering effect compared to the reference as shown in Fig. 2.

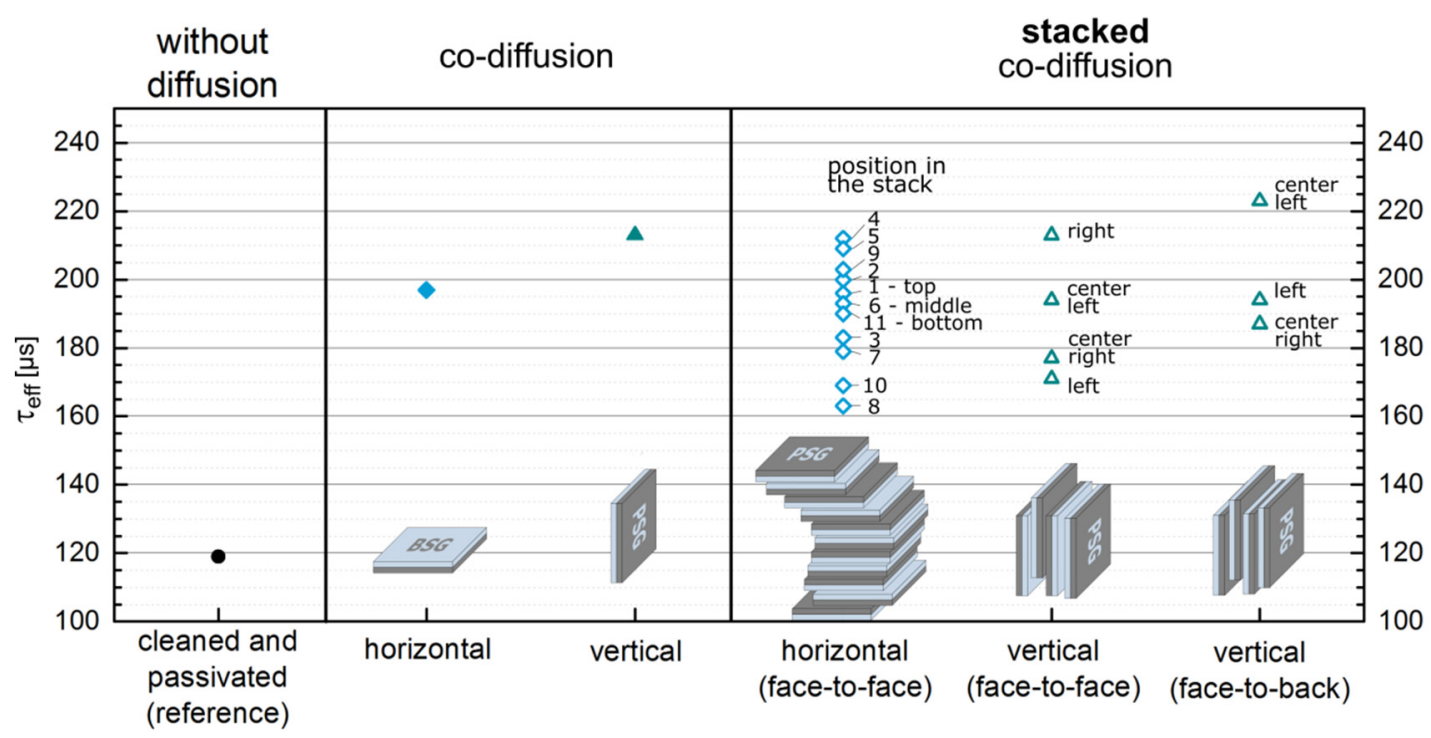

FIGURE. 2. Lifetime values (harmonic average) of reference, standard co-diffused and stacked co-diffused samples. The position within the horizontal stack of 11 wafers does not play a role for the lifetime improvement. Gettering in face-to-face and face-to-back vertical stacked co-diffusions is similarly effective. The right hand side sample of the face-to-back diffused stack got lost during sample processing.

All samples which underwent a stacked co-diffusion also show significantly higher $\tau_{\text {eff }}$ than the reference (Fig. 2). The average $\tau_{\text {eff }}$ of the 11 horizontally stacked co-diffused samples amounts to $\tau_{\text {eff }}=190 \mu$ s, which is comparable to the non-stacked horizontally diffused sample with $\tau_{\text {eff }}=197 \mu \mathrm{s}$. The worst horizontally stacked codiffused sample still improved by more than $40 \mu \mathrm{s}$. In addition, no correlation between the position within the stack and the gettering efficacy was observed.

For the horizontal stack and the vertical face-to-face stack, every second wafer was flipped to avoid a direct contact between BSG and PSG of neighbouring wafers. The comparison between the vertical face-to-face stack and vertical face-to-back stack shows similar results which means that flipping was not necessary (note that BSG and PSG were capped with $20 \mathrm{~nm} \mathrm{SiO} y: H)$. The average $\tau_{\text {eff }}$ of the vertically stacked co-diffused samples hardly differs from horizontal-stacked co-diffused samples and amounts to $\tau_{\text {eff }}=194 \mu \mathrm{s}$. The standard (vertically) APCVD glasses based co-diffused sample has $\tau_{\text {eff }}=213 \mu$ s after gettering.

Considering that stacking of samples above each other for co-diffusion potentially alters the temperature distribution within the wafers, different gettering efficacy in inner and outer parts of the samples could occur. For this reason $\tau_{\text {eff }}$ was measured spatially resolved (Fig. 3). The only cleaned and passivated reference sample reveals a rather non-homogeneous distribution of lifetimes. Without gettering several large areas with low lifetimes are found in midst of regions with moderate lifetimes (Fig. 3, top left). After co-diffusion gettering, large contiguous areas with significantly improved lifetimes are found and only a few low lifetime regions are still present. The $\tau_{\text {eff }}$ maps of stacked co-diffused samples do not differ significantly from single slot co-diffused samples. Also no major differences between inner and outer parts of the stacked co-diffused samples are seen. 


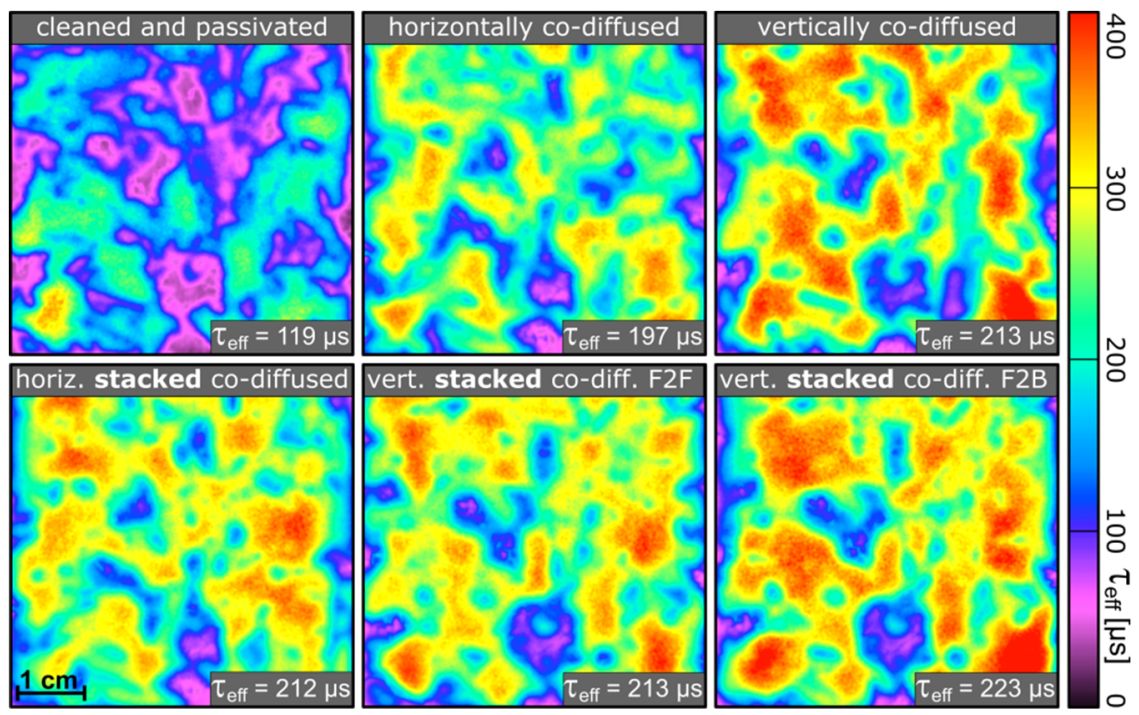

FIGURE. 3. Spatially resolved $\tau_{\text {eff }}$ measurements of reference, co-diffused and in different kinds of stacks co-diffused samples (exemplarily). All samples have similar grain and defect structures (sister samples) and a size of $5 \times 5 \mathrm{~cm}^{2}$. Harmonic average $\tau_{\text {eff }}$ values in each bottom right corner. F2F - face-to-face orientation; F2B - face to back orientation.

In order to separate the gettering efficacy of APCVD BSG and PSG in the co-diffusion, some samples were BSG / PSG coated on both sides and also diffused in stacks of 11 wafers. Fig. 4 shows an overlay of the spatially resolved $\left[\mathrm{Fe}_{\mathrm{i}}\right]$ measurements with optical grain boundary measurements. The comparison of the only cleaned and passivated reference sample with the BSG diffused sample shows no notable $\mathrm{Fe}_{\mathrm{i}}$ gettering. In contrast, the both sided PSG diffusion was slightly more effective than the co-diffusion. Therefore, the $\mathrm{Fe}_{i}$ gettering in the co-diffusion can be ascribed to phosphorus diffusion gettering. Moreover, PSG on only one side of the sample is sufficient to (nearly) reach the gettering efficacy of the both sided stacked PSG diffusion. These findings are consistent with the non-stacked APCVD glasses based co-diffusion gettering results reported in [2].
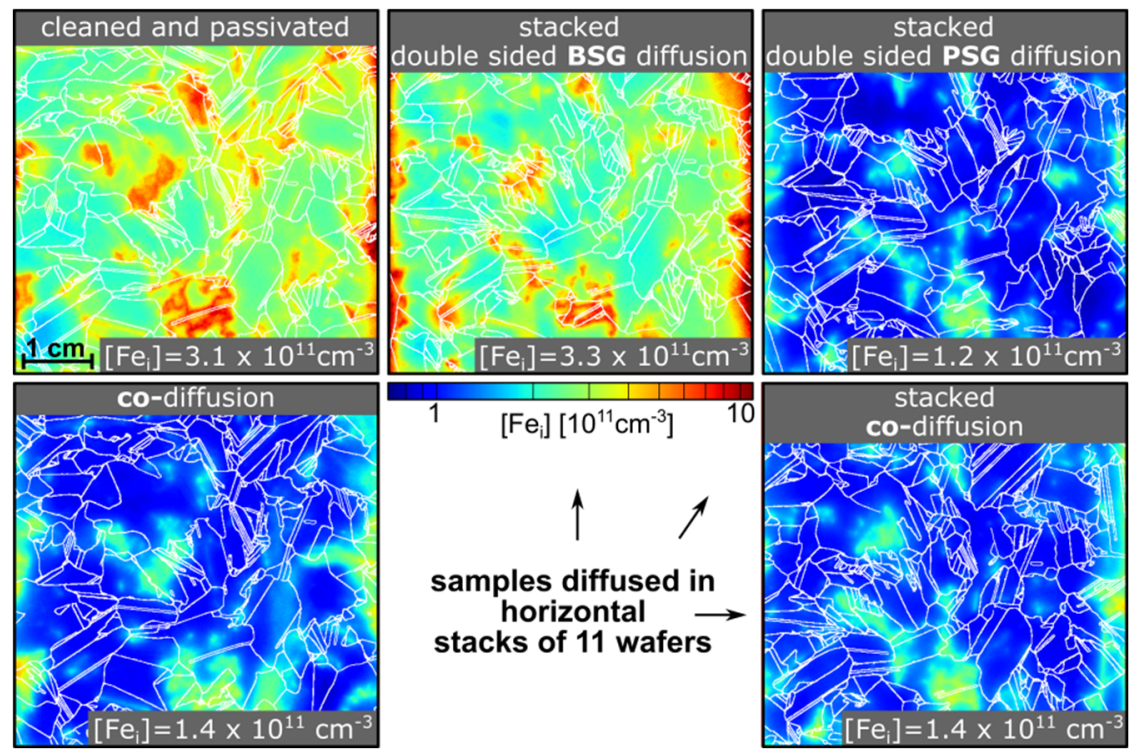

FIGURE. 4. Overlay of [ $\left.\mathrm{Fe}_{\mathrm{i}}\right]$ maps of reference, co-diffused and stacked co-diffused samples with optical grain boundary measurements (white). All samples have similar grain and defect structures (sister samples) and a size of $5 \times 5 \mathrm{~cm}^{2}$. Arithmetic average $\left[\mathrm{Fe}_{\mathrm{i}}\right]$ values in each bottom right corner. 


\section{SUMMARY}

APCVD glasses based stacked co-diffusion gettering is nearly as effective as single slot co-diffusion gettering for p-type mc-Si. The stacked co-diffusion gettering efficacy cannot completely keep up with single slot codiffusion gettering but is certainly close. The diffusion from APCVD PSG is responsible for the observed $\mathrm{Fe}_{\mathrm{i}}$ gettering. BSG diffusion was not able to reduce $\mathrm{Fe}_{i}$ notably. However, APCVD glasses based co-diffusion gettering and APCVD PSG diffusion gettering are comparably effective. The different thermal history of the stacked samples is expected to be the reason for the differences. The gettering efficacy in stacked co-diffusion can probably be improved by a slight adjustment of the diffusion temperature profile. Altogether, the gettering results of the stacked co-diffusion were found to be sufficient to start a further experiment in which stacked co-diffused p-type mc-Si PERT solar cells will be produced.

\section{ACKNOWLEDGMENTS}

The authors would like to thank H. Zunft from Schmid Group and L. Mahlstaedt and J. Rinder from the photovoltaics division of the University of Konstanz for their support. Part of this work was funded by the German BMWi (0324041B and 0324001). The content is the responsibility of the authors.

\section{REFERENCES}

1. A. W. Blakers, A. Wang, A. M. Milne, J. Zhao, and M. A. Green, Appl. Phys. Lett. 55, 1363-1365 (1989).

2. J. Fichtner, H. Zunft, A. Zuschlag, H. Knauss, and G. Hahn, IEEE J. Photovolt., 8(6), 1464-1469 (2018).

3. D. Kiliani, A. Herguth, G. Micard, J. Ebser, and G. Hahn, Sol. Energy Mat. Sol. Cells 106, 55-59 (2012).

4. G. Zoth and W. Bergholz, J. Appl. Phys. 67, 6764-6771 (1990).

5. D. H. Macdonald, L. J. Geerligs, and A. Azzizi, J. Appl. Phys. 95, 1021-1028 (2004).

6. D. Macdonald, J. Tan, and T. Trupke, J. Appl. Phys. 103,073710 (2008). 